\title{
Spatial orientation of galaxies in the core of the Shapley concentration - the cluster Abell 3558
}

\author{
B. Aryal $^{1,2}$, S. M. Kandel ${ }^{2}$, and W. Saurer ${ }^{1}$ \\ 1 Institut für Astrophysik, Universität Innsbruck, Technikerstraße 25, 6020 Innsbruck, Austria \\ e-mail: [binil.aryal; walter.saurer]@uibk.ac.at \\ 2 Central Department of Physics, Tribhuvan University, Kirtipur, Kathmandu, Nepal \\ e-mail: sunilkandel80@yahoo.com
}

Received 10 March 2006 / Accepted 15 May 2006

ABSTRACT

\begin{abstract}
We present an analysis of the spatial orientations of 323 galaxies in the cluster Abell 3558. Our aim is to examine non-random effects in galaxy orientations in the subsample of the cluster. The "position angle-inclination" method is used to find the threedimensional rotation axes. To check for anisotropy or isotropy we carried out three statistical tests: Fourier, Kolmogorov-Smirnov, and Kuiper-V. We assumed a spatially isotropic distribution to examine non-random effects. The galaxies found in the investigated cluster region show isotropy in both two- and three-dimensional analyses of the galactic coordinate system. In addition, it is found that the overall isotropic appearance cannot be maintained when looking at the subsamples. The low radial-velocity galaxies in A 3558 showed anisotropy in both two- and three-dimensional analyses. A weak anisotropy is noticed in the hard X-ray emission region. Few subsamples shows similar alignments with respect to both the galactic and Supergalactic coordinate systems. We offer possible explanations of these anisotropies.
\end{abstract}

Key words. galaxies: clusters: general - galaxies: evolution - galaxies: spiral - galaxies: distances and redshifts galaxies: formation - galaxies: statistics

\section{Introduction}

The Shapley Concentration is the richest supercluster within a distance of $300 h^{-1} \mathrm{Mpc}\left(h=H_{0} / 100\right)$. The core of the Shapley Concentration is a chain formed by the ACO (Abell et al. 1989) clusters A 3558, A 3562, A 3556 and by the two poor clusters SC 1327-312 and SC 1329-313. The main cluster A 3558, also known as Shapley 8 , is the richest cluster listed in the ACO catalogue, the only one with a richness class 4 . Within a circle of 1 Abell radius $\left(\sim 1.5 h^{-1} \mathrm{Mpc}\right)$ around this cluster, about 267 galaxies with measured redshifts in the velocity range $10260-18516 \mathrm{~km} \mathrm{~s}^{-1}$ are given in catalogues, so this cluster complex is one of the best-sampled galaxy clusters in the catalogue. The cluster A 3558 is in fact the most evolved system in the group, termed as a early-type or B-M type I (Bautz \& Morgan 1970) cluster. These clusters have systematically higher X-ray luminosities than other types (Ledlow et al. 2003). Cooling flows are found in a large fraction of them because of the presence of a cD galaxy and a correspondingly deeper gravitational potential (McHardy 1978). These clusters are dynamically very active and are still in the process of forming from their parent clusters or vice versa.

A substructure analysis (Bardelli et al. 1998) briefly discusses the core of the Shapley Concentration and suggests that the core complex is the result of a series of incoherent groupgroup and cluster-group mergings. Venturi et al. (2000) studied radio properties in connection with the dynamical state of the cluster Abell 3558 complex and found that the ratio between the radio and the optical flux for the Shapley radio galaxies is not affected by the local galaxy density. This result suggests that the high optical density in the cluster has no effect on the radio galaxy emissivity. The core of the Shapley Concentration is dynamically very active (Bardelli et al. 1994), and the main cluster A 3558 appears to be interacting with the other clusters, A 3562 and A 3556; it is therefore very interesting to study the spatial distribution of angular momentum in the cluster galaxies. Because spatial orientations of the angular momentum of disk galaxies can indicate the initial conditions when galaxies and clusters formed. The study of the orientations of the rotation axes of cluster galaxies is very important for understanding the process of formation of this large structure.

Many theories use this distributions to develop scenarios. The "Pancake model" by Doroshkevich (1973), the "Hierarchy model" by Peebles (1969), and the "Primordial vorticity model" of Ozernoy (1978) predict different scenarios concerning the origin of angular momentum of galaxies in clusters. Thus, studies of the angular momentum distribution of galaxies in clusters may explore important ideas in order to understand galaxy evolution scenarios. The "pancake model" (see, e.g. Doroshkevich 1973; Shandarin 1974; Doroshkevich \& Shandarin 1978) predicts that the rotation axes of galaxies tend to lie within the cluster plane. According to the "hierarchy model" the directions of the rotation axes should be distributed randomly. The "primordial vorticity theory" predicts that the rotation axes of galaxies are primarily distributed perpendicular to the cluster plane.

We use the "position angle - inclination" method (Flin \& Godlowski 1986) to compute three-dimensional spatial orientation vectors from two-dimensional measured parameters. Their method has been applied by several authors in galaxy orientation studies (Flin 1988; Kashikawa \& Okamura 1992; Godlowski 1993, 1994; Hu et al. 1995, 1998; Wu et al. 1997; Yuan et al. 1997; Godlowski et al. 1998; Godlowski \& Ostrowski 1999; 
Flin 2001; Aryal \& Saurer 2004, 2005a,b,c, 2006; Baier et al. 2003; etc.). Their method, not only analyzed the distributions of galaxy PAs, but also considered another important parameter - the galaxy's inclination with respect to the observer's line of sight. These two angles allowed us to find the orientations of the two possible vectors normal to the galactic plane, one of them assumed to be the galactic rotation axis or spin vector of the galaxy.

Aryal \& Saurer (2004) studied the spatial orientation of 296 galaxies in A 3558 and found anisotropy in both the twodimensional (equatorial PA-distribution) and three-dimensional analysis (polar and azimuthal angle distributions). In the threedimensional analyses, they found that the spin vector (SV hereafter) orientation of galaxies tends to lie parallel to the Local Supercluster plane and that SV projections of galaxies tend to be directed towards the Local Supercluster center (or Virgo cluster center). In this paper, we intend to find the local anisotropies in A 3558 and explain the role of such anisotropies in the merging environment (within the cluster A 3558) and in the clustercluster collision environment (with other clusters A 3556 and A 3562).

Kitzbichler \& Saurer (2003) studied the Coma cluster, where they noticed local anisotropies in the X-ray emission region and concluded that the X-ray subcluster of the Coma cluster might be falling in on the main cluster. We are interested in studying the preferred alignments of galaxies in hard X-ray emission region of the cluster A 3558 in order to test a possible coincidence of hot-gas features with the local anisotropy.

Godlowski et al. (2003) describe a relation between angular momentum and the mass of a galaxy and tested this relation for Tully's galaxy group (Godlowski et al. 2005). A random galaxy alignment for that group is noticed. They conclude that the minima of the relation between angular momenta and masses of galaxy structures can be verified observationally.

We expect to carry out the following studies: (1) study the preferred alignment of the rotation axes of galaxies in the cluster with respect to the galactic coordinate system; (2) test the existence of a morphological, magnitudinal, diameter, and radial velocity (RV) dependence in the galaxy orientation; (3) investigate the emission region in other wavelengths and study the preferred alignment in that particular region; and, finally, (4) see what else we can say about the origin of angular momenta from this study.

This paper is organized as follows. In Sect. 2 we describe the sample used and the method of data reduction. We give the results of a multiwavelength study in Sect. 3. In Sect. 4 we describe the methods, statistical tools and the selection effects. Finally, a discussion of the statistical results and the conclusions are presented in Sects. 5 and 6.

\section{The sample}

We inspected all galaxies found in the cluster A 3558 region on the film copies (red-sensitive ESO) with the aid of a binocular microscope. The galaxies in the cluster were not too faint and it was relatively easy to determine diameters and position angles of many galaxies with relatively good accuracy. The galactic contamination in and around the clusters was minimum. A list of the data for A 3558 cluster as given in the ACO catalogue is given in Table 1.

To locate the cluster region on the films, the coordinates of the cluster center as given in the ACO catalogue was used. A circular survey area was defined using a radius $R=90^{\prime \prime}(1+z)^{2} / z$ (Thompson 1976). Here $z$ represents the redshift of the cluster. The foreground field galaxies in the investigated cluster
Table 1. Data of the cluster A 3558 as given in the ACO (Abell et al. 1989) catalogue.

\begin{tabular}{ll|ll}
\hline \hline Parameters & Properties & Parameters & Properties \\
\hline RA (2000) & $13^{\mathrm{h}} 27^{\mathrm{m}} 54^{\mathrm{s}} 0$ & $R V\left(\mathrm{~km} \mathrm{~s}^{-1}\right)$ & 14390 \\
Dec (2000) & $-31^{\circ} 29^{\prime} 00^{\prime \prime}$ & B-M type & $\mathrm{I}$ \\
$l$ & $311.99^{\circ}$ & R-C type & $\mathrm{R}$ \\
$b$ & $30.73^{\circ}$ & $m$ & 15.1 \\
$L$ & $148.87^{\circ}$ & $R$ & 4 \\
$B$ & $-1.37^{\circ}$ & $D$ & 3 \\
\hline
\end{tabular}

region were identified with the help of the Uppsala General Catalogue of Galaxies (Nilson 1973, UGC hereafter), the Third Reference Catalogue of Bright Galaxies (de Vaucouleurs et al. 1991), the ESO/Uppsala Survey of the ESO (B) Atlas (Lauberts 1982, ESO hereafter), the Morphological Galaxy Catalogue (Vorontsov-Vel'Yaminov et al. 1962-74; MGC hereafter), and the Southern Galaxy Catalogue (Corwin et al. 1985). These field galaxies have been removed from the database.

Aryal \& Saurer (2004) have roughly estimated the number of background galaxies in the cluster region on the basis of the area distribution of background galaxies around the cluster region. The number of background galaxies per unit area around the cluster region is calculated and divided by the number of galaxies per unit area in the cluster region. They estimated that the background contamination of galaxies in the investigated cluster region is $\approx 12 \%$.

The method of investigation and the measurements of positions, PAs, and diameters $(a, b)$ of galaxies were the same as in Aryal \& Saurer (2004). We measured positions (using DSS images with the help of the software ESO/ECF/CADC, Association of Universities for Research in Astronomy, Inc., version 1.3), diameters (using a transparent scale with units of one tenth of a millimeter), and PAs (using a protractor of $15 \mathrm{~cm}$ radius and a transparent glass with a thin line) for the investigated galaxies in the cluster region. We investigated 366 galaxies in the Abell 3558 cluster region. The diameters and position angle was measured for $366(100 \%)$ and $323(88.3 \%)$ galaxies. In the NED (NASA Extragalactic Database, http://nedwww.ipac.caltech.edu), the radial velocities of $217(59.3 \%)$ galaxies were listed until the cutoff date (September 2005). Our database includes 53 (16.4\%) spiral and $\mathrm{S} 0$ galaxies.

We studied the distribution of the apparent diameters of the galaxies to get the knowledge of the completeness of the data. For this, a constant spatial density for the galaxies with equal linear diameter was assumed. It was expected that the logarithm of the total number of galaxies having an apparent diameter greater or equal to a given limit is increasing with a slope of -3 . As indicated in Fig. 2a, the optical search is complete down to a diameter of $1^{\prime}$ (corresponding to $a=0.85 \mathrm{~mm}$ on the filmcopies). We calculated 83 and 240 galaxies up to the search limit (SL) and the below limit (BL). The histograms of the axial ratio $(b / a)$, magnitude, and the morphological distribution are shown in Figs. 2b-d. The lack of nearly face-on galaxies can be seen in Fig. 2b. The spiral galaxies are found to be dominated by S0 galaxies in A 3558. The radial velocity distribution of galaxies can be seen in Fig. 3. About $75 \%$ of the galaxies in A 3558 have RVs in the range 13000 to $16000 \mathrm{~km} \mathrm{~s}^{-1}$.

The cluster position angle is estimated assuming that the galaxies in the cluster are approximately equidistance. A straight line is fitted using the least-square fit method for the total galaxy, as well as for galaxies having $a \geq 1^{\prime}, a \geq 45^{\prime \prime}, a \geq 30^{\prime \prime}$ in the 


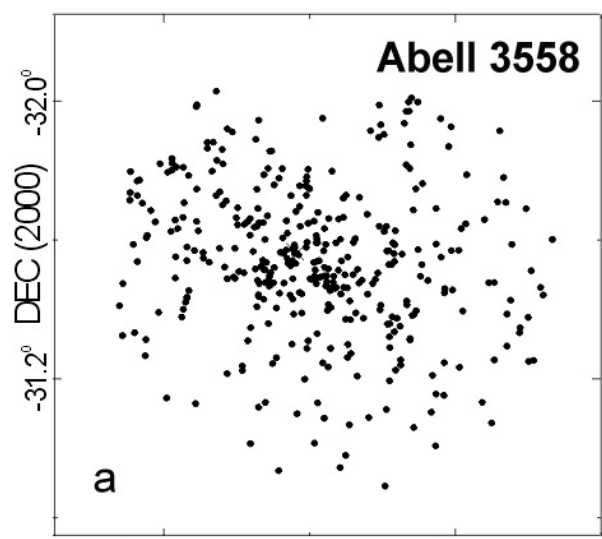

$13^{\mathrm{h}} 29^{\mathrm{m}} 07^{\mathrm{s}}$ RA (2000) $13^{\mathrm{h}} 26^{\mathrm{m}} 06^{\mathrm{s}}$
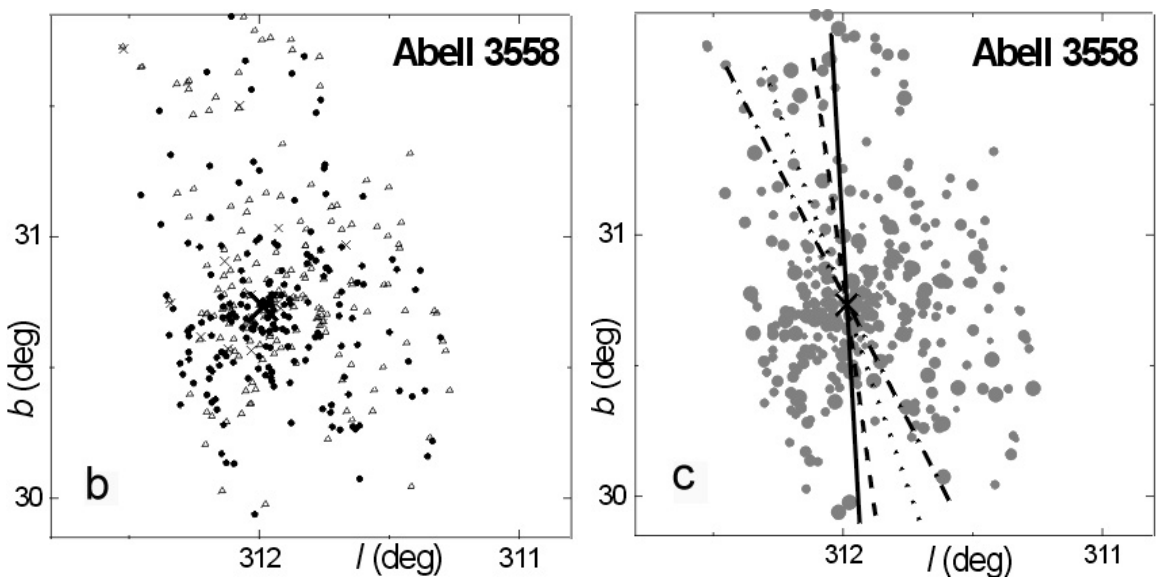

Fig. 1. The positions of 366 galaxies investigated in the cluster A 3558 region in the equatorial coordinate system a) and galactic coordinate system b), c)). The cross in the figure represents the cluster center. The symbol " $\triangle$ ", " $\times$ " and "•" represent the galaxies with unknown PA, unknown RVs and known PA and RV, respectively b). In Fig. 1c, four different sizes of the dots represent the major diameters $a \geq 1^{\prime}, 1^{\prime}<a \leq 45^{\prime \prime}$, $45^{\prime \prime}<a \leq 30^{\prime \prime}$ and $a \leq 30^{\prime \prime}$ according to their sizes, respectively. The solid, dashed, dot, and dash-dot line represent the best-fitted straight lines (using the least-square fit method) for the galaxies having major diameters $a \geq 1^{\prime}, a \geq 45^{\prime \prime}$, all, and $a \geq 30^{\prime \prime}$, respectively c).
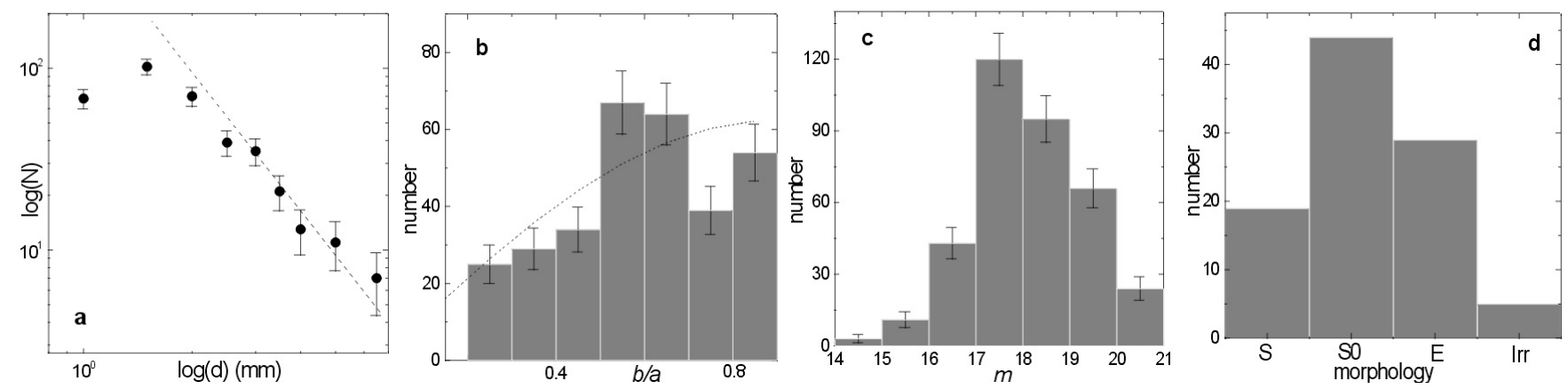

Fig. 2. a) Logarithm of the total number of galaxies greater than a given diameter versus the logarithm of this very diameter. The solid line represents the theoretical line having slope -3 . The axial ratio $(a / b)$ and the magnitude $(m)$ distribution of galaxies in A 3558 is shown in Figs. $3 b$ and $3 \mathrm{c}$. The histogram of the morphological classification of galaxies is shown $\mathbf{d}$ ).

Table 2. Major diameter statistics. The first two columns give the samples and the number of galaxies. The next three columns give the mean value, standard deviation $( \pm \mathrm{sd})$, and standard error $( \pm \mathrm{se})$ of the galaxy samples for $a \geq 1^{\prime}, a \geq 45^{\prime \prime}, a \geq 30^{\prime \prime}$, and all. The last column gives the cluster position angle (cPA) in the galactic coordinate system. The cPA is estimated using the least-square fit method (see Fig. 1c).

\begin{tabular}{llllll}
\hline \hline Samples & $N$ & Mean & \pm sd & \pm se & cPA \\
\hline$a \geq 1^{\prime}$ & 52 & $1.50^{\prime}$ & $23^{\prime \prime}$ & $3.9^{\prime \prime}$ & $3^{\circ}$ \\
$a \geq 45^{\prime \prime}$ & 126 & $69^{\prime \prime}$ & $26^{\prime \prime}$ & $2.3^{\prime \prime}$ & $7^{\circ}$ \\
$a \geq 30^{\prime \prime}$ & 298 & $48^{\prime \prime}$ & $25^{\prime \prime}$ & $1.4^{\prime \prime}$ & $22^{\circ}$ \\
all & 366 & $42^{\prime \prime}$ & $25^{\prime \prime}$ & $1.3^{\prime \prime}$ & $18^{\circ}$ \\
\hline
\end{tabular}

cluster region. The statistics of the samples are given in Table 2. The standard deviation and standard error is at a minimum for the total galaxies. The cPA for the all galaxies is estimated to be $18^{\circ}$ north-west.

We classified these galaxies into 7 subsamples according to their morphologies, radial velocities, diameters, and magnitudes, as follows: (1) spirals and S0 galaxies: $\mathrm{S}+\mathrm{S} 0$; (2) radial velocity in the range $13000<\mathrm{RV}\left(\mathrm{km} \mathrm{s}^{-1}\right) \leq 14250$ : $\mathrm{RV} 1$; (3) radial velocity in the range $14250<\mathrm{RV}\left(\mathrm{km} \mathrm{s}^{-1}\right) \leq 16000: \mathrm{RV} 2$; (4) $a \geq 1^{\prime}$ : SL (search limit); (4) $a<1^{\prime}$ : BL (below limit) (6) magnitude $(m): 17<m \leq 18$ (M1); and (7) magnitude $(m)$ : $18<m \leq 19$ (M2).
We classified the radial velocity and magnitude on the basis of the minimum standard deviation. The subsamples RV1 (M1) and RV2 (M2) showed approximately identical standard deviation values (within their standard error limit). The center $\mathrm{cD}$ galaxy $\left(\mathrm{PA}=166^{\circ}\right)$ falls into the subsamples RV1, M1, and SL.

\section{Multiwavelength study}

We used SkyView (http://skyview.gsfc.nasa.gov/) to search for counterparts to the investigated cluster region (the cluster A 3558). The SkyView Virtual Observatory gives hard $\mathrm{X}$-ray, soft X-ray, infrared, optical, UV, and radio surveys. We inspected 21 surveys focusing on our region of interest (RA (J2000): $13^{\mathrm{h}} 27^{\mathrm{m}} 54^{\mathrm{s}} .0$, Dec. (J2000): $-31^{\circ} 29^{\prime} 00^{\prime \prime} ; 2^{\circ} \times 2^{\circ}$ ). No emission is noted in the following surveys: HEAO 1 A-2 (Shafer 1983; Allen et al. 1994), INTEGRAL/SPI Survey of the Galactic Centre (20-40 keV), Rossi X-ray Timming Explorer (RXTE), All Sky Slew Survey (XSS) (Revnivtsev et al. 2004), HRI Survey, ROSAT PSPC, ROSAT All Sky Survey (RASS3), ROSAT All Sky Survey (Snowden et al. 1995), Two Micron All Sky Survey (2MASS), COBE Diffuse Infrared Background Experiment (DIRBE) (Hauser et al. 1990), SFD Dust Maps (Schlegel et al. 1998), The H-alpha Full Sky Map (Finkbeiner 2003), The Southern H-Alpha Sky Survey Atlas (SHASSA) (Gaustad et al. 2001), Near Earth Asteroid Tracking (NEAT) / SkyMorph Survey, Sloan Digital Sky Survey (SDSS), 34.5 MHz 

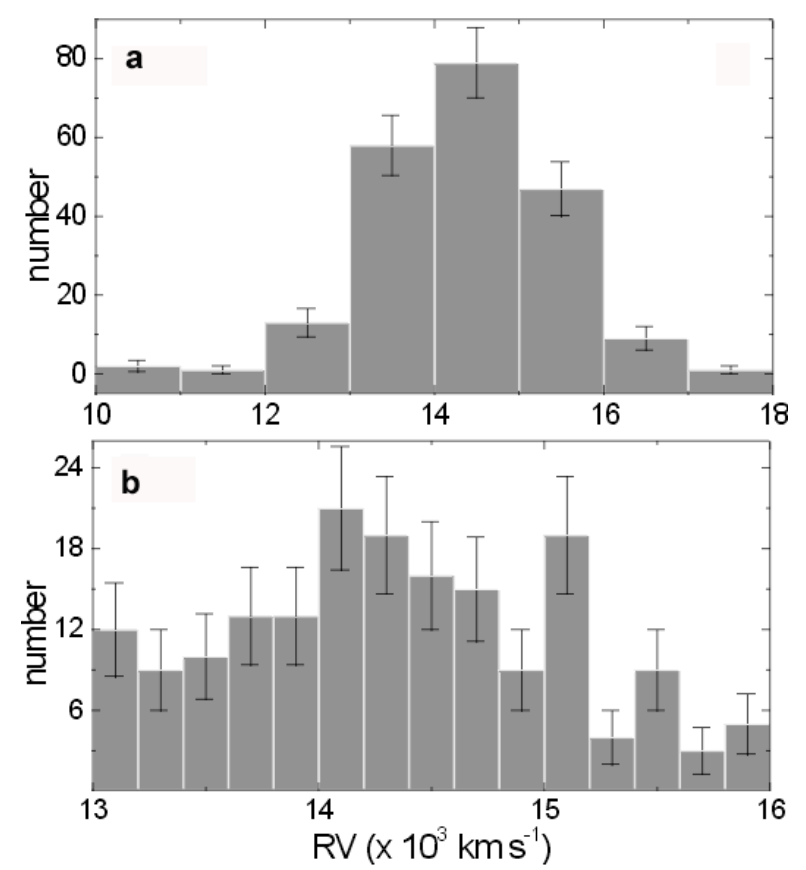

Fig. 3. The radial velocity distribution of galaxies in the cluster A 3558 region.

(Dwarakanath \& Udaya Shankar 1990), 408 MHz (Haslam et al. 1982), VLA FIRST Survey, NRAO VLA Sky Survey (NVSS), $4850 \mathrm{Mhz}$ and $4850 \mathrm{Mhz}$ (OLD) (Condon et al. 1993, 1991). We found a noticeable emission in two surveys: the GRANAT/SIGMA hard X-ray survey (40-100 keV) and $100 \mu \mathrm{m}$ IRAS survey.

A significant emission can be found in the GRANAT/SIGMA survey of hard X-ray sky (40-100 keV) (Paul et al. 1991; Revnivtsev et al. 2004) in the A 3558 cluster region (see Fig. 4a). Similarly, we found a significant $100 \mu \mathrm{m}$ infrared emission in IRAS Survey (Wheelock et al. 1991) in our region of interest (Fig. 4b).

Figure $4 \mathrm{a}$ displays $2^{\circ} \times 2^{\circ}$ hard X-ray $(40-100 \mathrm{keV})$ contour maps overlayed on an image centered at A 3558. In this figure, one notices that i) strong emission is found in the central region; ii) the maxima of the central emission is not at the cluster center; iii) the central emission is $\sim 0.4^{\circ}$ east of the cluster center; iv) the emission is more or less rounded; and v) there is no connection between the central emission and the surrounding weak-emission region. The hard X-ray contours span 11.5 to $23 \mathrm{MJy} / \mathrm{str}$ in steps separated by $2.3 \mathrm{MJy} / \mathrm{str}$. A total number of 216 galaxies are found in this emission region. A study of the spatial orientation of galaxies in this central emission region could be important. Any preferred alignment of galaxies in this region might indicate the coincidence of the hot-gas feature with optical condensations. We classify the galaxies found in this region as the 8th subsample (abbreviated as HX) for the alignment study.

In the IRAS survey, the emission is discernible not only at $100 \mu \mathrm{m}$, but also at $60 \mu \mathrm{m}$. Figure 4b shows $1^{\circ} \times 1^{\circ} 100 \mu \mathrm{m}$ HIRES contour maps (resolution $\sim 1^{\prime}$ ) projected on the image. The contours span 1.15 to $4.75 \mathrm{MJy} / \mathrm{str}$ in steps separated by $0.60 \mathrm{MJy} / \mathrm{str}$. In Fig. 4b, three emission regions are seen towards northeast. These emissions seem to be connected. The total number of galaxies found in this region is only 38. As we know that the IRAS sky region is relatively nearby sky and the cluster A 3558 is a distant cluster. Are these chance superpositions?

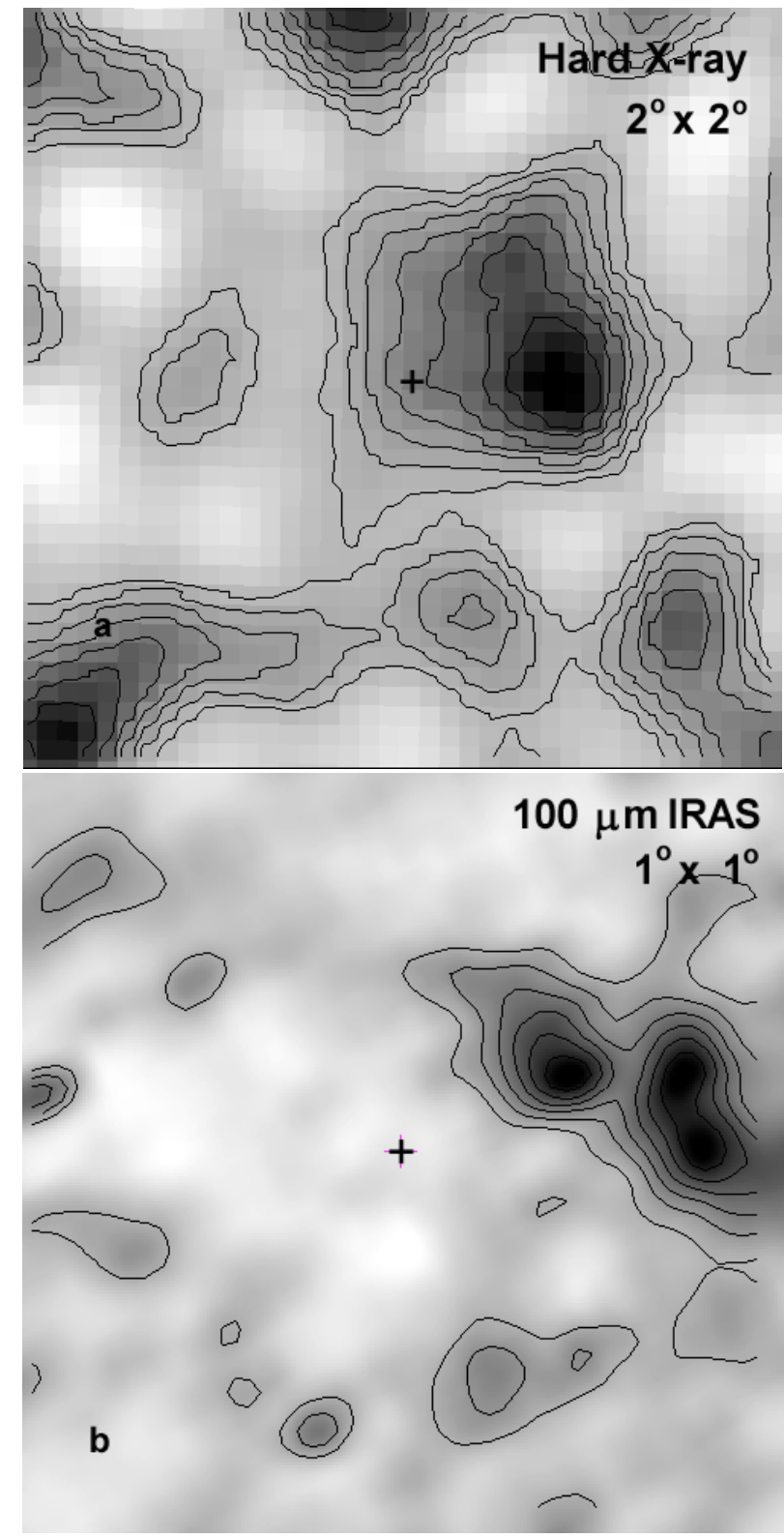

Fig. 4. Significant emission in the investigated cluster region in a) the GRANAT/SIGMA hard X-ray (40-100 keV) and b) the $100 \mu \mathrm{m}$ IRAS survey. The symbol "+" represents the center coordinate of the investigated cluster A 3558 as given in ACO catalogue. We used SkyView (http://skyview.gsfc.nasa.gov/) for these images. The hard X-ray contours a) span 11.5 to $23 \mathrm{MJy} / \mathrm{str}$ and $100 \mu \mathrm{m}$ IRAS contours b) span 1.15 to $4.75 \mathrm{MJy} / \mathrm{str}$ in steps separated by 2.3 and 0.6 , respectively.

Unfortunately there is no convincing way to find this out, since a lot of the structure in the IRAS maps exists on different scales. One of us (BA) has started a study to address this problem of superpositions on IRAS maps, although here we study the preferred alignment of galaxies found in this region (9th subsample - abbreviated as IR).

\section{Method of analysis}

We first give the method to find SVs of galaxies in Sect. 4.1. A brief introduction of the statistics is given in Sect. 4.2. Finally, the selection effects are described in Sect. 4.3. 


\subsection{Spin vector orientation}

We followed the usual method (see e.g. Flin \& Godlowski 1986) to derive the spatial orientations of SVs of galaxies. The threedimensional orientation of the SV of a galaxy is characterized by two angles: the polar angle $(\theta)$ between the galactic SV and a reference plane, and the azimuthal angle $(\phi)$ between the projection of a galactic SV on this reference plane and the $X$-axis within this plane. When using the galactic coordinate system as reference, $\theta$ and $\phi$ can be obtained from measurable quantities as follows:

$\sin \theta=-\cos i \sin b \pm \sin i \sin p \cos b$

$\sin \phi=(\cos \theta)^{-1}[-\cos i \cos b \sin l+\sin i$

$(\mp \sin p \sin b \sin l \mp \cos p \cos l)$

where $i$ is the inclination angle, estimated with Holmberg's (1946) formula: $\cos ^{2} i=\left[(b / a)^{2}-q^{2}\right] /\left(1-q^{2}\right)$ with $b / a$ the measured axial ratio. The values $l, b, p$, and $q$ represent the galactic longitude, latitude, position angle, and flatness parameter of galaxy, respectively. One major problem with applying the Holmberg model is the unique flatness parameter that is used for all types of galaxies. We used the flatness factor as suggested by Haynes \& Giovanelli (1984) for morphologically identified galaxies. They investigated the visual underestimation of the intrinsic axial ratio with the decreasing inclination that can be found in the major and minor diameters of the UGC and suggest different flatness factors for different types of galaxies, ranging from $q=0.23$ for ellipticals (E) and lenticulars (S0) to $q=0.10$ for late-type spirals (Scd, Sd). Morphologically unidentified galaxies dominate $(\sim 85 \%)$ our database. We used $q=0.20$ for these morphologically unidentified galaxies.

There is no information from which we can define a physically-based reference frame for the cluster A 3558. The reference plane is complex because of the merging and collisional environment in the cluster A 3558, so we used the galactic plane (i.e., the plane of the Milky Way) as a reference for the analysis.

In the galactic coordinate system, the principal axis is the galactic equator (the intersection of the plane of the Milky Way with the celestial sphere) and the reference points are the north galactic pole and the zero point on the galactic equator; the coordinates of a celestial body are its galactic longitude and latitude. In this system, the zero point on the galactic equator has the equatorial coordinates RA $(\mathrm{J} 2000)=17^{\mathrm{h}} 39.3^{\mathrm{m}}$ and $\operatorname{Dec}(\mathrm{J} 2000)=-28^{\circ} 55^{\mathrm{m}}$; this lies in the direction of the center of our galaxy, the Milky Way.

To calculate the SV orientation $(\theta, \phi)$ of a given disk galaxy, the measured quantities $\alpha, \delta$, PA (equatorial system) have to be transformed to the galactic variables $l, b, p$. We used the NED coordinate calculator for this transformation.

It is evident that, for a given value of $i$, the expressions (1) and (2) give two solutions for both $\theta$ and $\phi$ and hence 4 solutions for the angular momentum vector of a galaxy. For a large sample of galaxies, however, it is hardly possible to determine - for each galaxy - which one is physically correct. For the statistical analysis we took each of these possibilities into account independently, as usual.

We assumed a spatial isotropic distribution of SVs of galaxies as a theoretical reference. This spatial reference distribution gives further reference distributions for the angles $\theta$ and $\phi$. As a next step, our observations was compared with these isotropic distribution curves in both $\theta$ and $\phi$. For this comparison we used three different statistical tests: Fourier test, KolmogorovSmirnov test, and Kuiper- $V$ statistics.
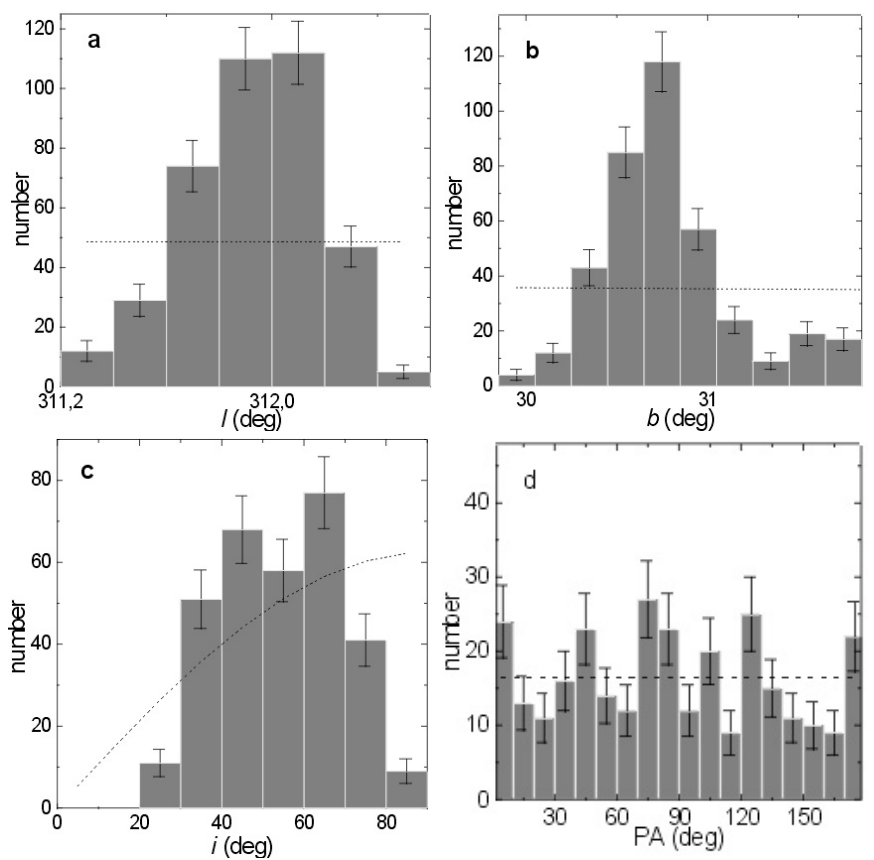

Fig. 5. The galactic longitude a), latitude b), inclination angle c) and equatorial position angle d) distribution of galaxies in A 3558 region. $\mathrm{PA}=0^{\circ}$ or $180^{\circ}$ corresponds to the galactic rotation axes lying in the galactic plane.

\subsection{Statistics}

We applied the Fourier (Godlowski 1993), K-S (Stephens 1970; Press et al. 1992; Kanji 1995) and Kuiper-V (Kuiper 1962; Stephens 1970) tests to the distribution of galactic position $(p)$, polar $(\theta)$, and azimuthal angle $(\phi)$. Note that these tests are a proper method in our case, because $p, \theta$ and $\phi$ are independent data. The significance level was chosen to be $95 \%$, as the null hypothesis is established to be an equidistribution for the $p, \theta$, and $\phi$. A spatial isotropy of the spin vectors was chosen as null hypothesis. In cases of too small a sample size, only the K-S and Kuiper-V tests are meaningful, because they can be applied to small samples, too.

\subsection{Selection effects and the error}

The inhomogeneous distributions of positions $(l, b)$ of galaxies in A 3558 can be seen in Figs. 5a,b. The humps at $312^{\circ}$ in $l$ and at $30.6^{\circ}$ in $b$-distributions are caused by the cluster center (Figs. 5a,b). In the axial ratio distributions, the decline at $0.70-0.90$ is due to the selection effect against nearly face-on galaxies. This effect can also be seen very clearly in the inclination angle distribution (Fig. 5c). No galaxies can be seen in the first bin $\left(i<10^{\circ}\right)$, and the number of galaxies in the first three bins is less than expected (Fig. 5c). The decline in the number of galaxies in the last two bins is probably due to the faint edge-on galaxies that could not be included in the database. Hence, we noticed three kinds of selection effects in our database: (1) inhomogenous distribution of positions of galaxies; (2) lack of knowledge of PAs of nearly face-on galaxies; and (3) lack of edge-on galaxies. These are thus the main selection effects.

As was shown by Aryal \& Saurer (2000, 2001), any selection criteria imposed on the data may cause severe changes in the shapes of the expected isotropic distribution curves. The inhomogeneous distribution of galaxy positions and the lack of our knowledge of the PAs of nearly face-on galaxies are the 
main selection effects. Because our galaxy samples are taken from the limited region of the sky, it is important to remove both the positional and the inclination effects. To do this we use the method described by Aryal \& Saurer (2000). In their method, a true spatial distribution of the galaxy rotation axis is assumed to be isotropic. Then, due to the projection effects, $i$ can be distributed $\propto \sin i, b$ can be distributed $\propto \cos b$, the variables $l$ and $p$ can be distributed randomly, and formulae (1) and (2) can be used to calculate the corresponding values of $\theta$ and $\phi$. The isotropic distribution curves are based on calculations including $1 \times 10^{6}$ virtual galaxies. These isotropic distribution curves in $\theta$ (Fig. 7) and $\phi$ (Fig. 8) are compared with the observations.

Due to poor knowledge of the real shapes of galaxies (Godlowski \& Ostrowski 1999; Godlowski et al. 2005) in the cluster, it is difficult to get correct theoretical distributions concerning the spatial orientation of galaxies. We believe that the relation between angular momenta and masses of galaxy structures in A 3558 gives the clue needed for the preferred alignment. This should be verified in the future.

To remove the Holmberg effect we adopted the method of Fouque \& Paturel (1985) in order to convert the measured diameters to standard photometric diameters. For this aim we measured the diameters of 78 galaxies for which diameters were given in the catalogue and compared those values with the measurements. The ratio of the measured and given diameters is found to be 1.07 to 1.12 (7-12\% larger). We used an additive constant for the ESO, UGC, and MGC catalogues to reduce the visual diameters of galaxies to photometric ones. We found that our data need an additive constant 0.17 to the visual diameters to put them on the photometric system.

Both $\theta$ and $\phi$ are assigned to be functions of $b, l, i$, and $p$. The errors in the coordinates and PAs were determined by comparing the measured values with known values in the catalogues (UGC, ESO, and MGC) and the errors turned out to be less than $10^{\prime}$ and $\pm 10^{\circ}$. The error in major and minor diameters, mainly due to a limited step size, was estimated to be $5^{\prime}$. Using the standard tool of error propagation, the errors in $i, \theta$, and $\phi$ can be estimated to be $6^{\circ}, 12^{\circ}$, and $17^{\circ}$, respectively.

\section{Results}

We first give the results of two-dimensional analysis (galactic PA-distribution) and later discuss their significance in threedimentional analysis ( $\theta$ and $\phi$-distribution).

\subsection{Anisotropy in the position angle distribution}

Here we analyze the distribution of the PAs (i.e., the PA of the major diameter) of galaxies instead of the rotation axes. The rotation axis is perpendicular to the galactic planes therefore, the conditions for anisotropy are the same as for the $\theta$ and $\phi$-distributions. Only the sign of $\Delta_{11}$ is opposite: $\Delta_{11}<0$ indicates an excess of galaxies with the galactic plane parallel to the equatorial plane.

Figure 6 shows the $p$-distribution of galaxies in the total sample and subsample. The statistical parameters for the galactic position angle $(p)$ distribution of the total sample and subsamples are given in Table 3. The first three columns give the samples, the dips and humps in the galactic position angle $(p)$ distribution (Fig. 6). The next two columns give the results of Kolmogorov-Smirnov test and Kuiper-V test: a "0" denotes that the null hypothesis (isotropy) cannot be rejected at the chosen significance level, "1" designates that the null hypothesis can be
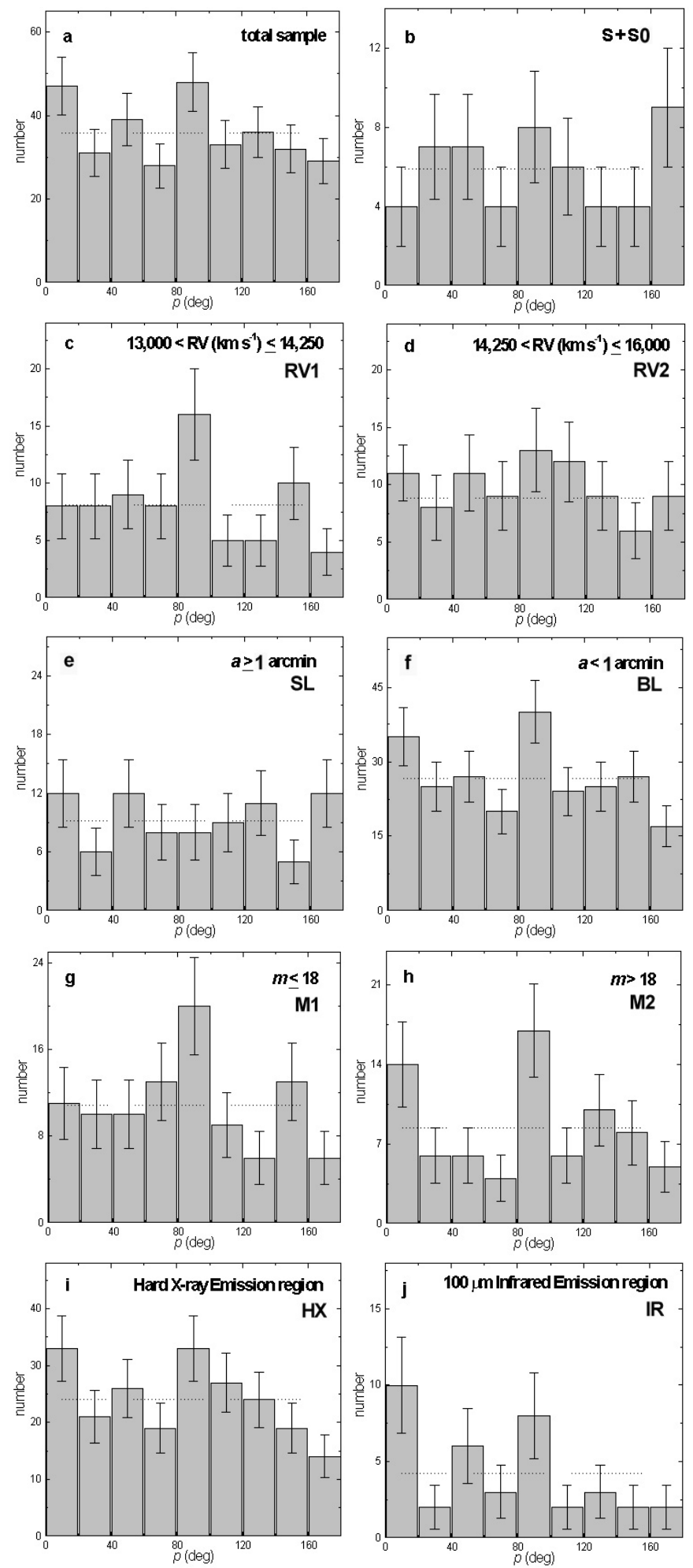

Fig. 6. The galactic position angle $(p)$ distribution of galaxies found in a) A 3558 region (total sample). The subsamples are: b) $\mathrm{S}+\mathrm{S} 0$ galaxies, c) galaxies with radial velocity in the range $13000-14250 \mathrm{~km} \mathrm{~s}^{-1}$ (RV1), d) galaxies with radial velocity $\geq 14250 \mathrm{~km} \mathrm{~s}^{-1}$ (RV2), e) galaxies up to the completeness of search limit (SL), f) galaxies below the search limit (BL), g) galaxies with magnitude $\leq 18$ (M1), h) galaxies with magnitude $>18$ (M2), i) galaxies found in the hard X-ray emission region (HX), and j) galaxies found in the $100 \mu \mathrm{m}$ IRAS emission region (IR). The observed counts with statistical $\pm \sigma$ error bars are shown. $\mathrm{PA}=0^{\circ}$ or $180^{\circ}$ corresponds to the galactic rotation axes lying in the equatorial plane. 
Table 3. Statistics of the galactic position angle distribution of galaxies in the total sample and subsamples.

\begin{tabular}{lccccccc}
\hline \hline Sample & Number & $\begin{array}{c}\text { Dips } \\
\text { (degree) }\end{array}$ & $\begin{array}{c}\text { Humps } \\
\text { (degree) }\end{array}$ & $\begin{array}{c}\text { K-S } \\
\text { test }\end{array}$ & $\begin{array}{c}\text { Kuiper V } \\
\text { test }\end{array}$ & $\begin{array}{c}\text { Fourier } \\
\Delta_{11} / \sigma\left(\Delta_{11}\right)\end{array}$ & $\begin{array}{c}\text { test } \\
P\left(>\Delta_{1}\right)\end{array}$ \\
\hline total & 323 & $70(\sim 1.5 \sigma)$ & $10,90(\sim 1.5 \sigma)$ & 0 & 0 & -0.381 & 0.813 \\
S+S0 & 53 & - & - & 0 & 0 & -0.028 & 0.837 \\
RV1 & 73 & $170(\sim 2 \sigma)$ & $90(\sim 2 \sigma)$ & 1 & 1 & -1.343 & 0.142 \\
RV2 & 88 & - & - & 0 & 0 & -1.075 & 0.266 \\
SL & 83 & - & - & 0 & 0 & +0.471 & 0.820 \\
BL & 240 & $170(\sim 2 \sigma)$ & $90(\sim 2 \sigma)$ & 0 & 0 & -0.719 & 0.661 \\
M1 & 98 & $130,170(>2 \sigma)$ & $90(2 \sigma)$ & 1 & 1 & -1.737 & 0.098 \\
M2 & 76 & $70(\sim 1.5 \sigma)$ & $90(>2 \sigma)$ & 0 & 0 & -0.419 & 0.704 \\
HX & 216 & $170(>2 \sigma)$ & $10,90(\sim 1.5 \sigma)$ & 0 & 0 & -1.227 & 0.207 \\
IR & 38 & - & $10(>1.5 \sigma)$ & 0 & 0 & -0.027 & 0.318 \\
\hline
\end{tabular}

rejected. The last two columns give the first-order Fourier coefficient $\left(\Delta_{11} / \sigma\left(\Delta_{11}\right)\right)$ and first-order Fourier probability $\left(P\left(>\Delta_{1}\right)\right)$.

The total sample shows the isotropy in all three statistical tests (Table 3). Similar to the total sample, the subsamples $\mathrm{S}+\mathrm{S} 0$, RV2, SL, BL, M2, HX and IR show no preferred alignment in the position angle distribution. Hence, a random orientation of PA of galaxies is found with respect to the galactic coordinate system.

The Kolmogorov-Smirnov and Kuiper-V tests show anisotropy in two subsamples: RV1 and M1. A significant hump at $90^{\circ}$ can be seen in these subsamples (Figs. 6c,g). In the Fourier test, the $P\left(>\Delta_{1}\right)$ is found $<15 \%$ in these subsamples. The $\Delta_{11}$ value is found at the $>1.3 \sigma$ level, suggesting that the galactic planes of the galaxies tend to lie parallel to the plane of the Milky Way, i.e., the galactic plane. In other words, the rotation axes of the galaxies tend to be oriented perpendicular to the galactic plane.

To understand the evolution of a cluster, a good knowledge of its reference system is needed. Using the least-square fit, we fitted a straight line for all galaxies in the investigated cluster region and estimated the cluster position angle as $18^{\circ}$. We studied the $p$-distributions of galaxies in the total sample and subsamples with respect to the estimated cluster PA. In the total sample, a hump at $\sim 10^{\circ}$ is noticed; however, it shows isotropy in the statistics. We do not notice any correlation between the estimated cluster position angle (i.e., $18^{\circ}$ ) and the humps or dips in the subsamples. No preferred alignment is noticed in the cluster position angle.

Hence, the total sample shows isotropy with respect to the cluster PA, as well as to the galactic plane. The low RV (RV1) and the low-magnitude (M1) galaxies in the cluster Abell 3558 show anisotropy. We compare our results of two-dimensional analysis ( $p$-distribution) and three-dimensional analysis ( $\theta$ and $\phi$ distribution) in Sect. 5.4.

\subsection{Anisotropy in the polar angle distribution}

Table 4 gives the statistical parameters and Fig. 7 shows this distribution in the galactic plane: $\theta=0^{\circ}\left(90^{\circ}\right)$ corresponds to the galactic SV and tends to lie parallel (perpendicular) the galactic plane. We assume weak anisotropy if, $1<\Delta_{11} / \sigma\left(\Delta_{11}\right) \leq 1.5$.

The total sample shows isotropy in all three statistics (Table 4). The expected isotropic distribution almost fits the observed distribution (Fig. 7a). A small dip at $57.5^{\circ}$ was not enough to change the statistical result to anisotropy. It should be noted that the dips at larger angles $\left(>45^{\circ}\right)$ and the humps at smaller angles $\left(<45^{\circ}\right)$ cause a negative $\Delta_{11}$ value, suggesting that the SVs of galaxies tend to lie parallel to the galactic plane. Here the $\Delta_{11}$ value is found well within the $\pm 1 \sigma$ error limit. Hence, no
Table 4. Statistics of the polar angle distribution of galaxies in the total sample and subsamples. See text for an explanation of the columns.

\begin{tabular}{lccccc}
\hline \hline Sample & number & K-S & Kuiper-V & $\Delta_{11} / \sigma\left(\Delta_{11}\right)$ & $P\left(>\Delta_{1}\right)$ \\
\hline total & 323 & 0 & 0 & -0.08 & 0.906 \\
S+S0 & 53 & 0 & 0 & -0.24 & 0.836 \\
RV1 & 73 & 1 & 1 & -1.25 & 0.203 \\
RV2 & 88 & 0 & 0 & -0.37 & 0.887 \\
SL & 83 & 0 & 0 & -0.38 & 0.814 \\
BL & 240 & 0 & 0 & -0.27 & 0.753 \\
M1 & 98 & 1 & 1 & +1.24 & 0.154 \\
M2 & 76 & 0 & 0 & -0.08 & 0.784 \\
HX & 216 & 0 & 0 & -0.83 & 0.460 \\
IR & 38 & 0 & 0 & -0.42 & 0.815 \\
\hline
\end{tabular}

preferred alignment is noticed in the spatial orientation of 323 galaxies found in the A 3558 cluster region.

In the $\theta$-distribution of the $\mathrm{S}+\mathrm{S} 0$ galaxies in $\mathrm{A} 3558$, both $\mathrm{K}-\mathrm{S}$ and Kuiper-V tests show isotropy. In these tests, the symbol " 0 " indicates that the null hypothesis cannot be rejected. In other words, our hypothesis (isotropy and homogeneous) is confirmed for this subsample. Here we do not discuss the results of the Fourier test because of poor statistics (number of the solution $<5$ in many bins, see Fig. $7 b$ ). Hence, we conclude a random orientation for $\mathrm{S}+\mathrm{S} 0$ galaxies.

Five humps at $17.5^{\circ}(>1 \sigma), 32.5^{\circ}(>1.5 \sigma), 37.5^{\circ}(>1 \sigma)$, $42.5^{\circ}(>1 \sigma)$, and $67.5^{\circ}(>1 \sigma)$ can be seen in the $\theta$-distribution of the galaxies with a radial velocity in the range $13000 \mathrm{~km} \mathrm{~s}^{-1}$ and $14250 \mathrm{~km} \mathrm{~s}^{-1}$ (RV1) (Fig. 7c). Four humps at smaller angles $\left(<45^{\circ}\right)$ cause a negative $\Delta_{11}$ value at $>1.2 \sigma$ level, suggesting that the SVs of low-RV galaxies tend to be aligned parallel to the galactic plane. The dips at $52.5^{\circ}(>1 \sigma)$ and $72.5^{\circ}(\sim 1 \sigma)$ support this result. The $\Delta_{11} / \sigma\left(\Delta_{11}\right)<1.5 \sigma$, because of dips at smaller angles $\left(<45^{\circ}\right)$. The first-order Fourier probability is found to be $20 \%$ because of these dips. Thus, the Fourier test shows weak anisotropy for this subsample. The results of the $\mathrm{K}-\mathrm{S}$ and Kuiper-V tests suggest anisotropy and thus that the null hypothesis can be rejected here. Hence, the SV orientation of RV1 galaxies in the cluster A 3558 tends to lie parallel to the galactic plane. All three statistical tests show isotropy in the subsample RV2 (Table 3). No significant hump can be seen in Fig. 7d. The low RV galaxies in the A 3558 region show a preferred alignment, whereas the high RV galaxies show a random alignment.

Similar to the subsamples RV1, the subsample M1 shows anisotropy in all three statistics. A hump at $82.5^{\circ}(>1.5 \sigma)$ and a dip at $2.5^{\circ}(>2 \sigma)$ make a positive $\Delta_{11}$ value at the $\sim 1.4 \sigma$ level in the subsample M1 (Table 3, Fig. 7g). Thus, the galaxies with lower magnitudes (mostly low-optical surface brightness 

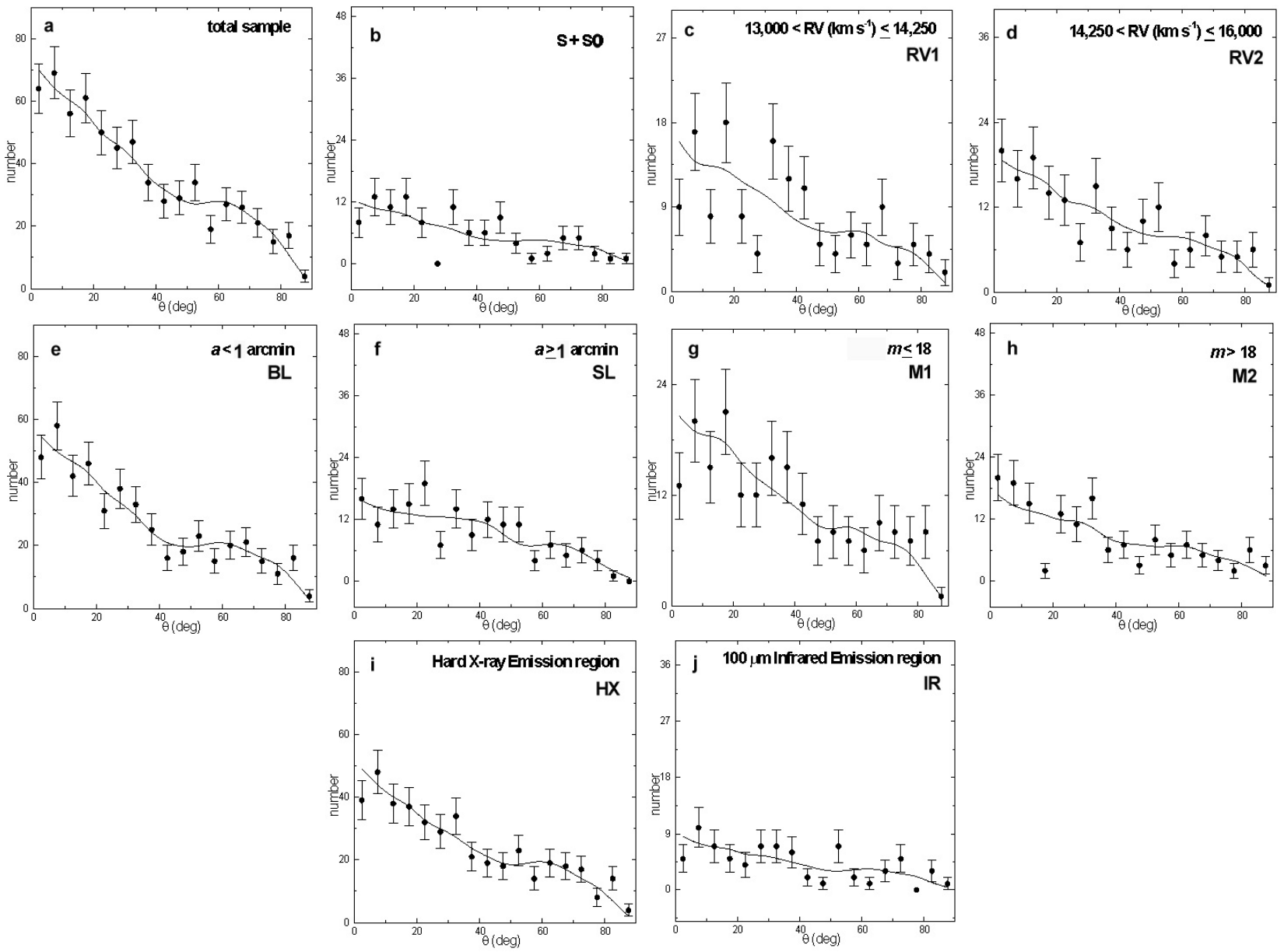

Fig. 7. The polar angle $(\theta)$ distribution in the galaxies found a) in the cluster A 3558 region (total sample). The subsamples are: b) S+S0, c) RV1, d) RV2, e) BL, f) SL, g) M1, h) M2, i) HX, and j) IR. These symbols are analogous to Fig. 6 . The observed counts with statistical $\pm 1 \sigma$ error bars are shown and $\theta=0^{\circ}$ corresponds to the galactic SV lying in the LSC plane. Anisotropy can be seen in the subsamples RV1 c) and M1 g).

galaxies) in the cluster A 3558 show that their SVs tend to be oriented perpendicular to the galactic plane. A random orientation is found for the galaxies having magnitudes greater than 18 .

No preferred alignment is shown by the galaxies found in both the hard X-ray and $100 \mu \mathrm{m}$ emission regions in the cluster field. All three statistics give isotropy in the subsamples HX and IR. A small dip at $2.5^{\circ}$ and humps at $22.5^{\circ}$ and $82.5^{\circ}$ were not enough to change the statistical result to show anisotropy in the subsample HX (Fig. 7i). We do not discuss the results of the Fourier test for subsample IR because of poor statistics (number of the solution $<5$ in many bins, see Fig. $7 \mathrm{j}$ ). We conclude isotropy for this subsample because of the results of the K-S and Kuiper-V statistics.

Thus, two subsamples RV1 and M1 show weak anisotropy in the polar angle distribution. The SVs of the low RV galaxies $\left(13000 \mathrm{~km} \mathrm{~s}^{-1}\right.$ to $\left.14250 \mathrm{~km} \mathrm{~s}^{-1}\right)$ in the cluster A 3558 tend to lie parallel to the galactic plane, whereas the low-magnitude galaxies $(m \leq 18)$ in the cluster A 3558 tend to be oriented perpendicular to the galactic plane. No preferred alignment of SVs of galaxies in the total sample and the subsamples S+S0, RV2, SL, BL, M2, HX, and IR is noticed.

\subsection{Anisotropy in the azimuthal angle distribution}

Statistical parameters for the azimuthal angle $(\phi)$ distribution of the total sample and subsamples are given in Table 5. Figure 8 shows the distribution of the SV projections $(\phi)$ of galaxies in the
Table 5. Statistics of the azimuthal angle distribution of galaxies in the total sample and subsamples. See text for an explanation of the columns.

\begin{tabular}{ccccc}
\hline \hline Sample & K-S & Kuiper-V & $\Delta_{11} / \sigma\left(\Delta_{11}\right)$ & $P\left(>\Delta_{1}\right)$ \\
\hline total & 0 & 0 & +1.01 & 0.495 \\
S+S0 & 1 & 1 & +1.58 & 0.103 \\
RV1 & 1 & 1 & +1.74 & 0.096 \\
RV2 & 0 & 0 & +1.07 & 0.427 \\
SL & 1 & 1 & +1.79 & 0.076 \\
BL & 0 & 0 & +0.15 & 0.820 \\
M1 & 0 & 0 & +0.46 & 0.607 \\
M2 & 0 & 0 & -0.52 & 0.560 \\
HX & 0 & 0 & +1.22 & 0.208 \\
IR & 0 & 0 & -0.23 & 0.855 \\
\hline
\end{tabular}

total sample and subsamples with respect to the galactic coordinate system. In the figures, $\phi=0^{\circ}$ means the direction towards the galactic center.

It is relatively easy to interpret the humps and dips in the histograms of the $\theta$-distribution. This is not the case with those of the $\phi$-distribution because of their ranges, $-90^{\circ}$ to $90^{\circ}$. In the histograms of the $\phi$-distribution, $\phi=0^{\circ}$ means the direction towards the center of the galactic coordinate system (e.g. Fig. 8). Humps in the region $-50^{\circ}$ to $+50^{\circ}$ and the dips at $-90^{\circ}$ to $-50^{\circ}$ (first 4 bins) and at $50^{\circ}$ to $90^{\circ}$ (last 4 bins) make the $\Delta_{11}$ value positive in the $\phi$-distribution. A positive $\Delta_{11}$ suggests that the $\mathrm{SV}$ projections of galaxies tend to point towards the galactic 

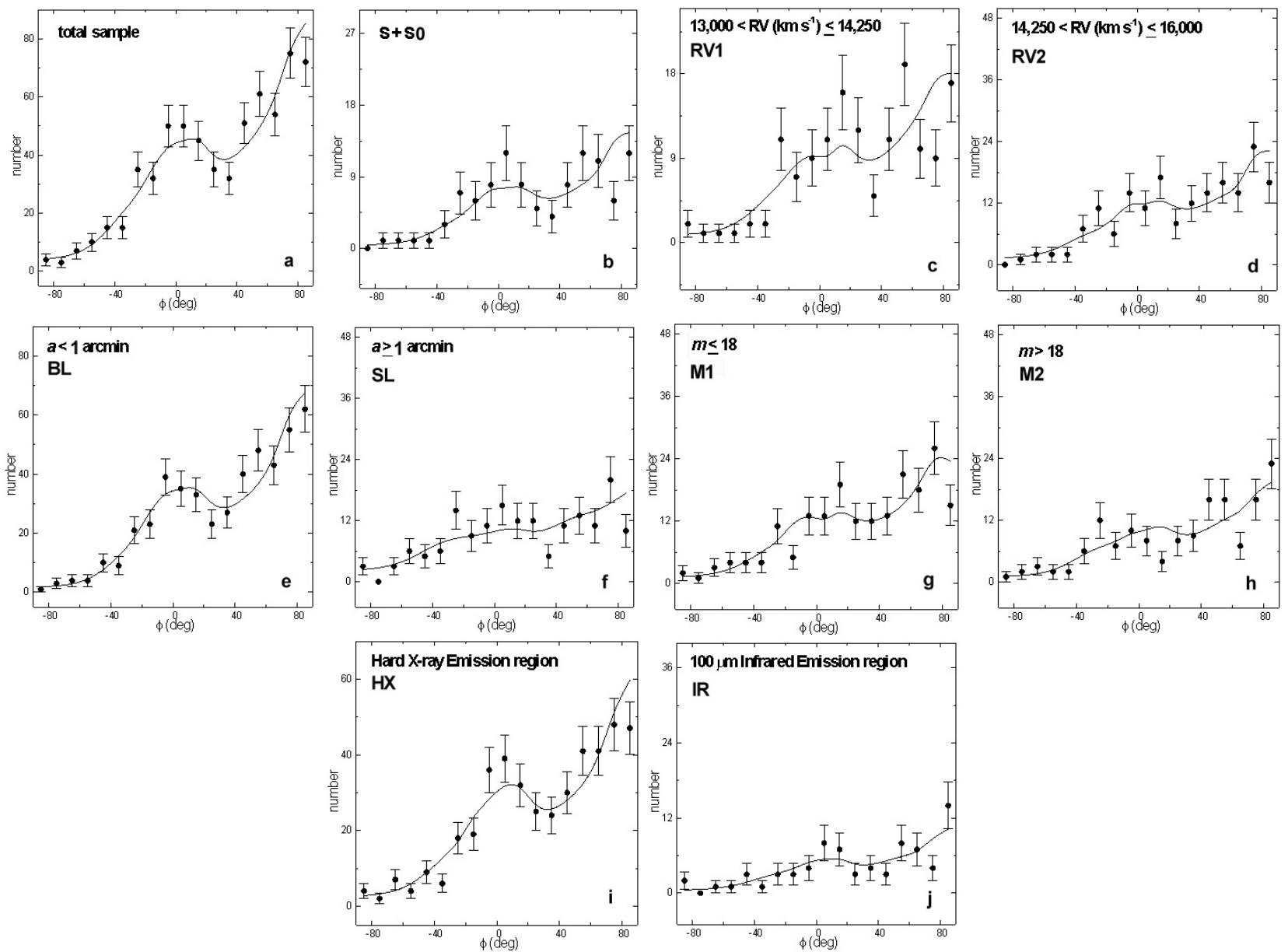

Fig. 8. The azimuthal angle $(\phi)$ distribution in a) the galaxies found in the cluster A 3558 region (total sample). The subsamples are: b) S+S0, c) RV1, d) RV2, e) BL, f) SL, g) M1, h) M2, i) HX, and j) IR. These symbols are analogous to Fig. 6 . The observed counts with statistical $\pm 1 \sigma$ error bars are shown, and $\phi=0^{\circ}$ means the direction to the galactic center. Anisotropy can be seen in the subsamples S+S0, RV1, and SL.

center. Similarly, a negative $\Delta_{11}$ suggests that the SV projections of galaxies tend to be oriented perpendicular with respect to the galactic center. In the $\phi$-distribution, the $\Delta_{11}$ value would be negative for $\phi$ when humps are at $-90^{\circ}$ to $-50^{\circ}$ (first 4 bins) and at $50^{\circ}$ to $90^{\circ}$ (last 4 bins) and dips are at $-50^{\circ}$ to $+50^{\circ}$.

Selection effects make the shape of the expected isotropic $\phi$-distribution curves rather complex (solid lines in Fig. 8). We ran simulations to examine the possible effects on the results of the Fourier test of the errors in $\phi\left(17^{\circ}\right)$. It turned out that the results of the Fourier test remains unchanged, so we regard the Fourier test as more reliable. We assumed weak anisotropy if $1<\Delta_{11} / \sigma\left(\Delta_{11}\right) \leq 1.5$.

The total sample shows isotropy in all three statistics (Table 5), meaning the distributions of SV projections of galaxies in the cluster A 3558 were found to be random. Similar to the total sample, the subsamples RV2, BL, M1, M2, and IR show isotropy in the $\phi$-distribution. The Fourier probability is $>40 \%$ in these samples. The results of the K-S and Kuiper-V tests for these samples give isotropy suggesting that the null hypothesis cannot be rejected here. Hence, no preferred alignment is found in the total sample as well as in the subsamples RV2, SL, M1, $\mathrm{M} 2$, and IR.

Anisotropy is found in the subsamples S+S0, RV1, and SL (Figs. 8b,c,f). This can be seen in the results of the statistics (Table 5). The $\Delta_{11}$ value is positive at $>1.5 \sigma$ level for these subsamples, suggesting that the SV projections of galaxies tend to point towards the center of the galactic coordinate system.
Thus, total sample and 6 subsamples show isotropy, and three subsamples ( $\mathrm{S}+\mathrm{S} 0, \mathrm{RV} 1$, and SL) show anisotropy in the $\phi$-distribution. These three subsamples show a similar tendency: the SVs projections of the galaxies tend to point towards the galactic center of the Milky Way.

\subsection{Discussion}

Aryal \& Saurer (2004) find anisotropy when analyzing 296 galaxies in A 3558 region in both the two-dimensional (equatorial PA-distribution) and three-dimensional analysis ( $\theta$ and $\phi$-distributions). In the three-dimensional analysis, they studied the preferred orientation with respect to the Supergalactic coordinate system and found that the SV of galaxies tend to lie parallel to the Local Supercluster plane and SV projections of galaxies tend to be directed towards the Local Supercluster center (Virgo cluster center). In this work, no preferred alignment was noticed in both the twoand three-dimensional analyze when analyzing 324 galaxies. Interestingly, we noticed a similar tendency to the one in Aryal \& Saurer (2004) for few subsamples of A 3558.

Figure 9 shows the orientation parameter (Fourier coefficient, $\left.\Delta_{11} / \sigma\left(\Delta_{11}\right)\right)$ plot for the position (a) polar (b) and azimuthal angle (c) of the total sample and subsamples. It can be seen in Fig. 9 that the general trend to alignment $\left(\Delta_{11}\right.$ is negative for PA and polar angle distributions; positive for azimuthal 

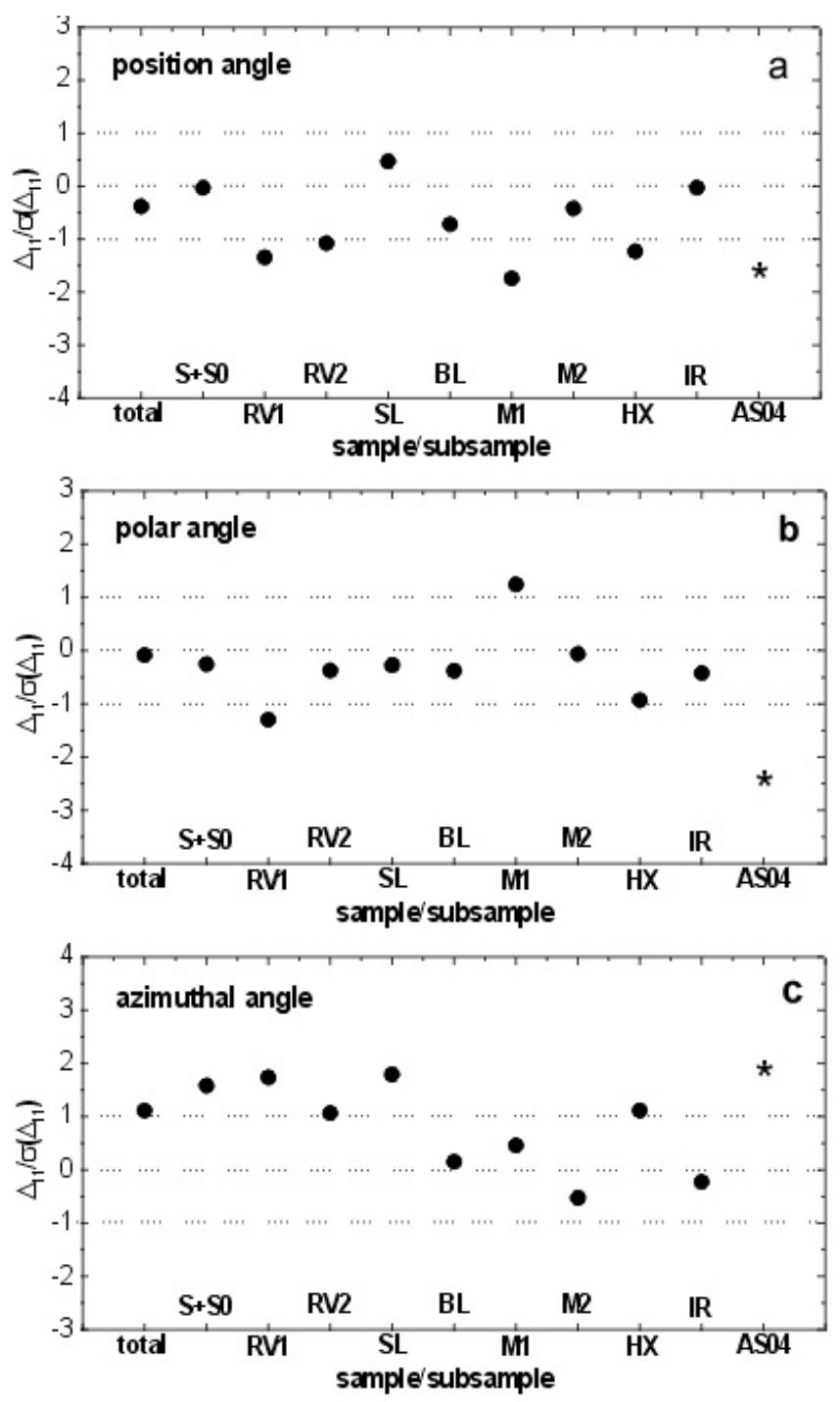

Fig. 9. The orientation parameter (the first-order Fourier coefficient, $\left.\Delta_{11} / \sigma\left(\Delta_{11}\right)\right)$ plot for a) position angle, b) polar angle $(\theta)$, and c) azimuthal angle $(\phi)$ of the total sample and subsamples. The symbol " $\star$ " represents the $\Delta_{11} / \sigma\left(\Delta_{11}\right)$ value obtained by Aryal \& Saurer (2004) for A 3558 with respect to the Supergalactic coordinate system. The region of isotropy and anisotropy is separated by the dashed lines: $-1 \leq \Delta_{11} / \sigma\left(\Delta_{11}\right) \leq+1$ for the isotropy and $\Delta_{11} / \sigma\left(\Delta_{11}\right)> \pm 1$ for anisotropy.

angle distributions) is found to be similar to both the galactic and Supergalactic systems.

The subsamples (total sample, RV2, SL, M2, HX, and IR) that showed isotropy in the two-dimensional analysis showed similar results in the three-dimensionl analysis. Thus, the projection effects in the two-dimensional analysis is studied better in the three-dimensional analysis.

The core of the Shapley Concentration is dynamically very active; the main cluster A 3558 appears to be interacting with the other clusters A 3562 and A 3556 (Bardelli et al. 1994). In a substructure analysis, Bardelli et al. (1998) briefly discuss the core of the Shapley concentration and suggest that the core complex is the result of a series of incoherent group-group and cluster-group mergings. Bardelli et al. (1998) studied the fraction of blue galaxies in the whole substructure and found that the bluest group is located between A 3558 and A 3562, i.e., in the expected position for the scenario of cluster-cluster collision.
Metcalfe et al. (1994) suggest that there may already have been an encounter between A 3558 and A 3562. In our study, high RV (14250 $\mathrm{km} \mathrm{s}^{-1}$ to $16000 \mathrm{~km} \mathrm{~s}^{-1}$ ) galaxies in A 3558 show random alignment. Thus, no preferred orientation is noticed in the bluest group of galaxies, suggesting that the cluster-cluster collision might be in an early phase or the collision environment has nothing to do with alignments of galaxies in A 3558 .

Venturi et al. (2000) carried out detailed statistical analysis of the radio properties in connection with the dynamical state of the A 3558 cluster complex and found that the ratio between the radio and the optical flux for the Shapley radio galaxies is not affected by the local galaxy density. It indicates that the high optical density in the cluster has no effect on the radio galaxy emissivity. Thus the merging environment plays no role in the radio power distribution of radio sources. Probably, this result could suggest that the merging environments have nothing to do with the random alignment of spin vector of galaxies in A 3558 with respect to the galactic coordinate system.

Kitzbichler \& Saurer (2003) studied the Coma cluster and suggest that there are deviations from a completely random galaxy orientation on small scales. They noticed local anisotropies in the X-ray emission region and concluded that the $\mathrm{X}$-ray subcluster of the Coma cluster might be falling in on the main cluster. We found that the galaxies found in the hard Xray emission region (subsample HX) in A 3558 show a weak anisotropy $\left(\Delta_{11}>1 \sigma\right)$ in the azimuthal angle distribution. This indicates a significant coincidence of hot-gas features identified in the hard X-ray observation (GRANAT/SIGMA Survey, Paul et al. 1991) of A 3558 with this local anisotropy.

The only subsample RV1 showed anisotropy in both the two and three-dimensional analyses. The centre $\mathrm{CD}$ galaxy (equatorial PA $=166^{\circ}$ ) belongs to this subsample. The spin vector orientations of low RV (13000 $\mathrm{km} \mathrm{s}^{-1}$ to $\left.14250 \mathrm{~km} \mathrm{~s}^{-1}\right)$ galaxies in A 3558 tend to lie parallel to the galactic plane and their SV projections tend to point towards the galactic center. This result might support the idea that the galaxies are falling in towards the centrally located $\mathrm{cD}$ galaxy.

We ran simulations to observe the changes in the results when the galactic plane of the Milky Way is rotated by an angle $\eta$. Our results for the subsample RV1 remain the same when $-20^{\circ} \leq \eta \leq+20^{\circ}$. This indicates that the low-RV galaxies in the A 3558 region support the pancake model, if the reference plane of the clusters does not deviate by more than $\pm 20^{\circ}$ from that of the galactic plane.

We compare our results for the subsample "S+SO" to Godlowski's (1993) subsample "S". He analyzed the S galaxies given in the UGC ESO Catalogue (Nilson 1973) and NGC Tully Catalogue (Tully 1988) separately. In the $\delta_{D}$-distribution $(\theta$ in our notation), he found isotropy for the spiral galaxies, similar to our result. He found anisotropy for the spirals (taken from the NGC Tully Catalogue) in the $\eta$-distribution ( $\phi$ in our notation). Interestingly, we obtained a similar result for the "S+S0" subsamples of the A 3558 although not at a very high significance level. Godlowski (1993) found that the SV projections of Spiral galaxies in the Local Supercluster tend to be oriented tangentially with respect to the Virgo cluster center. Godlowski (1994) used an identical sample. Obviously, the results for $\mathrm{S}$ in Godlowski (1994) are almost identical to those of Godlowski (1993). It is an interesting result that the spatial orientation of spiral galaxies in the nearby cluster (Local Supercluster, RV $\leq 3000 \mathrm{~km} \mathrm{~s}^{-1}$; Godlowski 1993) and the distant cluster (A 3558, RV $=\sim 14000 \mathrm{~km} \mathrm{~s}^{-1}$; present work) is identical to Supergalactic and galactic coordinate systems, respectively. It indicates that there is a good correlation 
between the physical reference plane of the investigated cluster and Local Supercluster plane, as well as the galactic plane of our Milky-way.

\section{Conclusion}

The galaxies investigated in the core of the Shapley Concentration, i.e., the cluster Abell 3558, shows isotropy in both the two- and three-dimensional analysis when analyzing with respect to the galactic coordinate system. Thus, our results for the total sample support the hierarchy model (Peebles 1969), which predicts that the directions of the spin vectors are entirely random. In addition, it is found that the overall isotropic appearance of galaxy orientation in A 3558 cannot be maintained when looking at the subsamples.

A few subsamples (RV1, M1 in $\theta$-distribution and $\mathrm{S}+\mathrm{S} 0$, $\mathrm{RV} 1, \mathrm{SL}$ in $\phi$-distribution) show anisotropy in the same direction, as concluded by Aryal \& Saurer (2004). The spin vector orientations of galaxies in these subsamples tend to lie parallel to the reference plane, and the spin vector projections tend to be directed towards the center of the reference system. This is an interesting result in that similar preferred alignments of galaxies in A 3558 is noticed with respect to both Supergalactic (Aryal \& Saurer 2004) and galactic coordinate systems (present study). Finding similar alignments with respect to the Supergalactic and galactic systems is a noticeable result. Probably, this result could indicate that the physical reference plane of the cluster might coincide with a plane of a higher concentration of galaxies at much larger scales.

The low-RV galaxies in A 3558 showed anisotropy in such a way that their spin-vector orientations tend to lie parallel to the galactic plane, and their spin vector projections tend to point towards the galactic center. This result supports the pancake model (Doroshkevich 1973; Shandarin 1974; Doroshkevich \& Shandarin 1978), which predicts that spin vectors of galaxies tend to lie within the cluster plane. A weak anisotropy is noticed in the hard X-ray emission region. We suspect that this result indicates an interesting coincidence of hot-gas features identified in the hard X-ray observation of A 3558 with this local anisotropy. We intend to work on this coincidence in the future.

Acknowledgements. The authors wish to thank the referee for the careful reading of the manuscript and the useful suggestions helped us improve the paper. This research has made use of the NASA/IPAC Extragalactic Database (NED), which is operated by the Jet Propulsion Laboratory, California Institute of Technology, under contract with the National Aeronautics and Space Administration, and the SkyView virtual observatory, operated at http://skyview.gsfc.nasa.gov/. We acknowledge Profs. Udayraj Khanal \& Mukunda Mani Aryal for their comments and suggestions. One of the authors (S.M.K.) acknowledges the Central Department of Physics, Tribhuvan University, Kirtipur, for providing various forms of support for his masters (M.Sc.) thesis.

\section{References}

Abell, G. O., Corwin, H. G., \& Olowin, R. P. 1989, ApJS, 70, 1 (ACO)

Abell, G. O. 1965, Ann. Rev. Astr. Ap., 3, 1

Allen, J., Jahoda, K., \& Whitlock, L. 1994, HEAO-1 and the A2 experiment, Legacy, 5

Aryal, B., \& Saurer, W. 2000, A\&A, 364, L97

Aryal, B., \& Saurer, W. 2001, in Galaxy Disk and Disk Galaxies, ed. J. S. Funes,

\& E. M. Corsini, ASP Conf. Ser., 230, 145

Aryal, B., \& Saurer, W. 2004, A\&A, 425, 871

Aryal, B., \& Saurer, W. 2005a, A\&A, 432, 841

Aryal, B., \& Saurer, W. 2005b, A\&A, 432, 431

Aryal, B., \& Saurer, W. 2005c, MNRAS, 360, 125

Aryal, B., \& Saurer, W. 2006, MNRAS, 366, 438
Baier, F. W., Godlowski, W., \& MacGillivray, H. T. 2003, A\&A, 403, 847

Bardelli, S., et al. 1994, MNRAS, 267, 665

Bardelli, S., Pisani, A., Ramella, M., Zucca, E., \& Zamoreni, G. 1998, MNRAS, 300,589

Bautz, L. P., \& Morgan, W. W. 1970, ApJ, 162, L149

Bukhari, F. A., \& Lawrence, E. C. 2003, A\&A, 283, 173

Condon, J. J., et al. 1993, BAAS, 25, 1389

Condon, J. J., Anderson, M. L., \& Helou, G. 1991, ApJ, 376, 95

Corwin, H. G., de Vaucouleurs, A., \& de Vaucouleurs, G. 1985, Univ. Texas Monogr. Astron., 4, 1

de Vaucouleurs, G., de Vaucouleurs, A., Corwin, et al. 1991, Third Reference Catalogue of Bright Galaxies (New York: Springer-Verlag)

Doroshkevich, A. G. 1973, ApJ, 14, L11

Doroshkevich, A. G., \& Shandarin, S. F. 1978, MNRAS, 184, 643

Dwarakanath, K. S., \& Udayashankar, N. 1990, A\&A, 211, 323

Finkbeiner, D. P. 2003, ApJS, 146, 407

Flin, P. 1988, MNRAS, 235, 857

Flin, P. 2001, MNRAS, 325, 49

Flin, P., \& Godlowski, W. 1986, MNRAS, 222, 525 (FG)

Fouque, P., \& Paturel, G. 1985, A\&A, 150, 192

Gaustad, J. E., McCullough, P. R., Rosing, W., \& van Buren, D. 2001, PASP, 113,1326

Godlowski, W. 1993, MNRAS, 265, 874 (Go93)

Godlowski, W. 1994, MNRAS, 271, 19

Godlowski, W., \& Ostrowski, M. 1999, MNRAS, 303, 50

Godlowski, W., Baier, F. W., \& MacGillivray, H. T. 1998, A\&A, 339, 709

Godlowski, W., Szydlowski, M., Flin, P., \& Biernacka, M. 2003, J. General Relativity \& Gravitation, 35, No. 5, 907

Godlowski, W., Szydlowski, M., \& Flin, P. 2005, J. General Relativity \& Gravitation, 37, 615

Haslam, C. G. T., Stoffel, H., Salter, C. J., \& Wilson, W. E. 1982, A\&AS, 47, 1

Hauser, M. G., Kelsall, T., Leisawitz, D., \& Weiland, J. 1990, COBE Ref. Pub. No. 97-A

Haynes, M., \& Giovanelli, R. 1984, AJ, 89, 6, 758

Holmberg, E. 1946, Medd. Lund. Astron. Obs., Ser VI, No. 117

Hu, F. X., Wu, G. X., Su, H. J., \& Liu, Y. Z. 1995, A\&A, 302, 45

Hu, F. X., Yuan, Q. R., Su, H. J., Wu, G. X., \& Liu, Y. Z. 1998, ApJ, 495, 179

Kanji, G. K. 1995, 100 Statistical tests, Sage Publication

Kashikawa, N., \& Okamura, S. 1992, PASJ, 44, 493

Kitzbichler, M. G., \& Saurer, W. 2003, ApJ, 590, L9

Kuiper, N. A. 1962, Proceedings of the Kononklijke Nederlandse Akademie Van Wetenschappen, Ser. A, 63, 38

Lauberts, A. 1982, ESO/Uppsala Survey of the ESO B Atlas. ESO, Garching bei Muenchen

Ledlow, M. J., Voges, W., Owen, F. N., \& Burns, J. O. 2003, AJ, 126, 2740

MacGillivray, H. T., McNally, B. V., Corwin, H. G., \& Dodd, R. J. 1982, MNRAS, 198, 605

McHardy, I. 1978, MNRAS, 184, 783

Metcalfe, N., Godwin, J. G., \& Peach, J. V. 1994, MNRAS, 267, 431

Nilson, P. 1973 Uppsala General Catalogue of Galaxies, Nova Acta Uppsala University, Ser. V.A., 1 (UGC)

Ozernoy, L. M. 1978, in The Large Scale Structure of the Universe, ed. M. S. Longair, \& J. Einasto, Proc. IAU Symp., No., 79 (Dordrecht: Reidel), 427

Paul, S. J., et al. 1991, AdSpR, 11, 289

Peebles, P. J. E. 1969, ApJ, 155, 393

Press, W. H., Teukolsky, S. A., Vetterlin, W. T., \& Flannery, B. P. 1992, Numerical Recipes in C, 2nd edn. (Cambridge University Press)

Revnivtsev, M., Sazonov, S., Gilfanov, M., \& Jahoda, K. 2004, A\&A, 418, 927

Schlegel, D. J., Finkbeiner, D. P., \& Davis, M. 1998, ApJ, 500, 525

Shafer, R. A. 1983, Spatial Fluctuations in the Diffuse X-Ray Background, University of Maryland, Ph.D. thesis

Shandarin, S. F. 1974, Astr. Zh 51,667 (in Russian), Soviet Astron 18, 392 (in English)

Snowden, M., et al. 1995, ApJ, 454, 643

Stephens, M. A. 1970, Journ. Royal Statistical Society, Ser. B, Vol. 32, 115

Thompson, L. A. 1976, ApJ, 209, 22

Tully, R. B. 1988, Nearby Galaxy Catalog. (Cambridge: Cambridge Univ. press)

Ventury, T., Bardelli, S., Morganti, R., \& Hunstead, R. W. 2000, MNRAS, 314, 594

Vorontsov-Vel, Y. B. A., Krasnogorskaya, A. A., \& Arkhipova, V. P. 1962-74, in Morphological Catalogue of Galaxies (Part I-V), Trudy Gosud. Astron. Inst. Shternberga (MGC)

Wheelock, M., et al. 1991, BAAS, 23, 908 (MGC)

Wu, G. X., Hu, F. X., Su, H. J., \& Liu, Y. Z. 1997, A\&A, 323, 317

Yuan, Q. R., Hu, F. X., Su, H. J., \& Huang, K. L. 1997, AJ, 114, 1308 